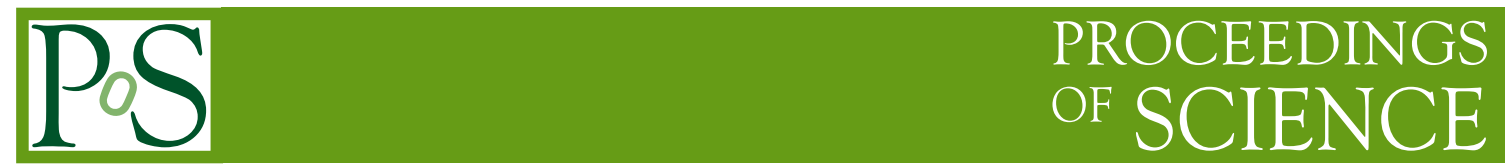

\title{
Status and recent highlights from ATLAS
}

\author{
Thorsten Wengler*t \\ CERN \\ E-mail: Thorsten.Wengler@cern.ch
}

The start-up of the ATLAS detector for 2016 data-taking is described, together with its current status and performance, and a selection of recent physics results is presented.

Fourth Annual Large Hadron Collider Physics

13-18 June 2016

Lund, Sweden

*Speaker.

† On behalf of the ATLAS collaboration. 


\section{ATLAS start-up in 2016 and overall detector performance}

The ATLAS experiment [1] has had a successful start of the 2016 data-taking run. Starting from April 12th, 2016, quiet beams were available in ATLAS, with the first stable beams declared on April 23rd. Since then the accelerator has ramped up the intensity and luminosity of the collisions, leading to $2.8 \mathrm{fb}^{-1}$ recorded by the time of this conference. The data-taking efficiency of the experiment with respect to stable beam collisions at this time was already $91 \%$, approaching the values of the 2015 run. The status of the detector is also excellent, with close to $100 \%$ of readout channels available across all sub-detectors.

The event selection and readout systems have been prepared for the expected 2016 conditions, with trigger selection menus up to instantaneous luminosities of $1.2 \times 10^{34} \mathrm{~cm}^{-2} \mathrm{~s}^{-1}$. As can be seen in Fig. 1, most of the bandwidth of the first level of the trigger is still given to generic triggers, such as single isolated leptons, complemented by multi-object triggers and triggers dedicated to specific analyses.

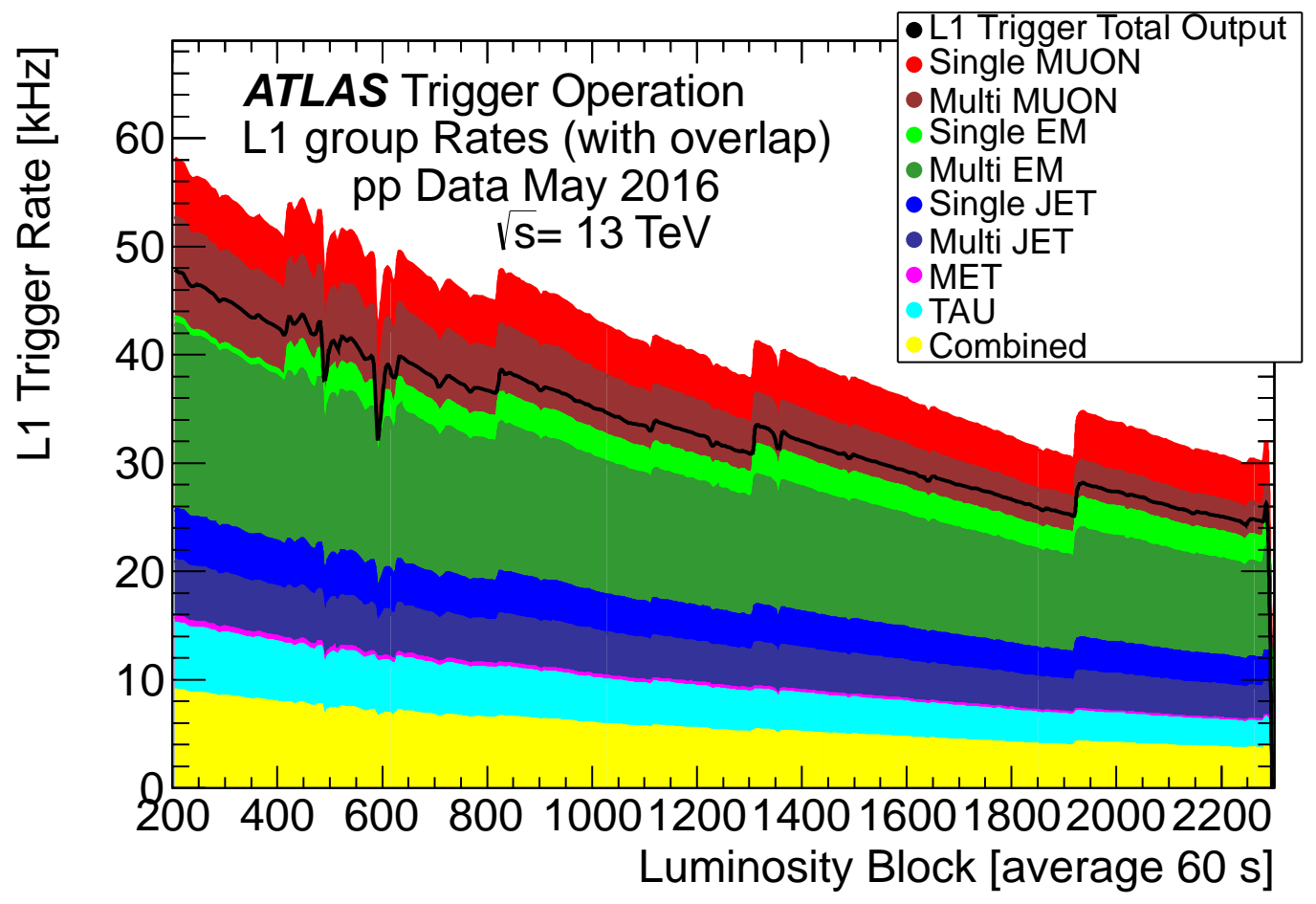

Figure 1: Physics trigger group rates at the first trigger level (L1) as a function of the luminosity block number. Each luminosity block correspond on average to 60s. The fill was taken in May 2016 with a peak luminosity of $3.6 \times 10^{33} \mathrm{~cm}^{-2} \mathrm{~s}^{-1}$ and an average number of interactions per beam crossing of $\sim 22$. Presented are the rates of the individual L1 trigger groups for various L1 trigger physics objects. Overlaps are accounted for in the total output rate, but not in the individual groups, leading to a higher recording rate compared to the total L1 output rate.

The inner detector, consisting of a silicon pixel detector, followed by a silicon strip detector and a transition radiation tracker has adopted well to the much higher instantaneous luminosities 
delivered by the machine in 2016. The transition radiation tracker has developed a number of gas leaks over the last years, leading to higher operation costs when running the whole detector with Xenon gas. A new gas configuration was introduced for 2016 along with a re-optimisation of the particle ID algorithms, that allow to run the detector partially with Argon instead of Xenon with negligible impact on physics. The silicon strip detector is working well, with nearly $99 \%$ of the modules fully operational. The readout of the pixel detector system, a limiting factor for the first level trigger rate in 2015, was upgraded for 2016 to handle the higher data rate. The alignment of the detector is already on the same level as in 2015, as can be seen for the innermost layer (IBL) of the pixel detector in Fig. 2 (left). The performance of the full tracking system is illustrated on the right of Fig. 2, showing the number of tracks associated to each primary vertex. The distribution is well modelled by the simulation, demonstrating a good understanding of the detector configuration.
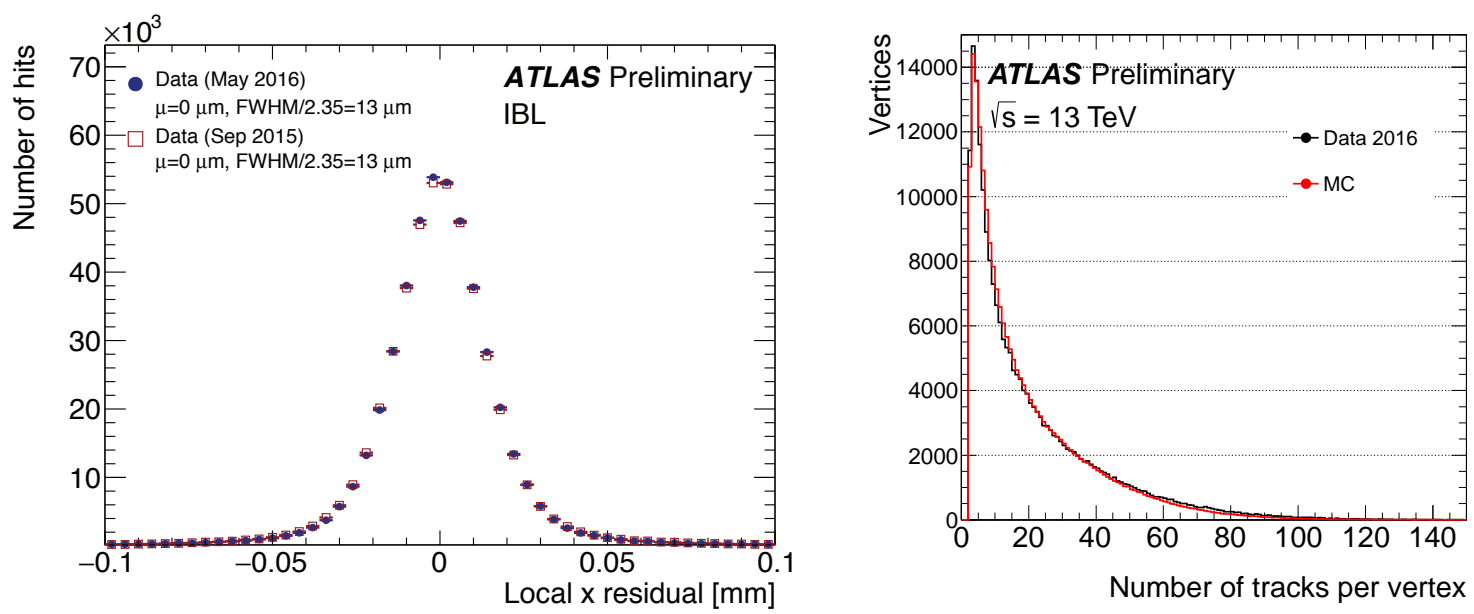

Figure 2: Left: The IBL local x unbiased residual distributions for 2015 data (red open squares) and for 2016 data (solid blue circles). The distributions are integrated over all hits-on-tracks in the IBL modules. The parameter $\mu$ represents the mean of the distributions. The distributions have been normalized to the same number of entries. The Full Width Half Maximum (FWHM) of the distributions is shown as a figure of merit. Right: The number of tracks associated to each reconstructed primary vertex, shown for the data from ATLAS run 298687 and for pile-up only Monte Carlo simulation. The Monte Carlo has been normalized to the data yield.

The muon spectrometer is working well for both precision tracking and triggering. Fig. 3 (left) shows the di-muon invariant mass obtained using the 2015 alignment on the 2016 data. The right side of Fig. 3 shows the muon trigger efficiency in the barrel region of the detector. A clear improvement is evident in the azimuthal region from about -1.5 to -2.2 , due to the installation of additional chambers in the feet region of the detector. Improvements have also been achieved on the endcap muon trigger system, where an optimisation of the coincidences between the detector layers have lead to a rate reduction of $8 \%$ for a muon trigger threshold of $20 \mathrm{GeV}$ without affecting the efficiency of the trigger.

Both Tile and LAr calorimeters are working smoothly. During the shutdown a small number of front-end electronics boards of the Tile calorimeter have been repaired, with all channels now operational. The LAr calorimeters are operating very stably, with excellent high voltage stability and LAr purity. The left side of Fig. 4 compares the reconstructed invariant mass distribution of 

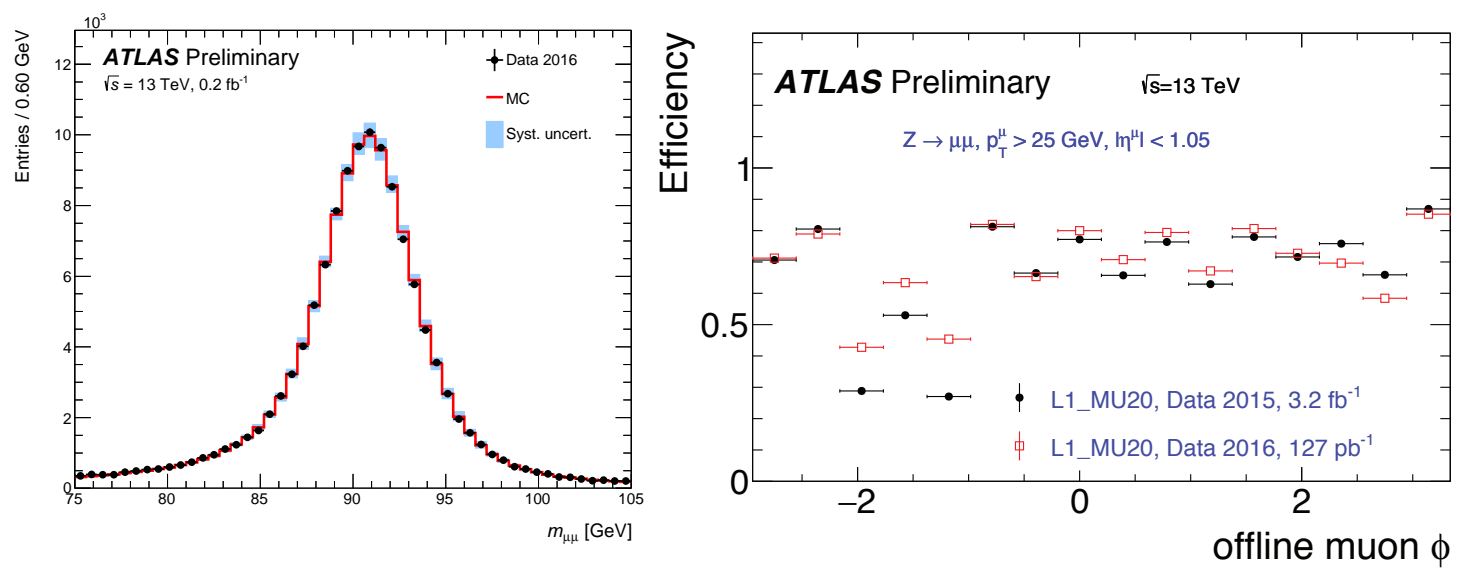

Figure 3: Left: Di-muon invariant mass distribution of $\mathrm{Z} \rightarrow \mu \mu$ events reconstructed in the inner detector and muon systems. The points show the data. The continuous line corresponds to the simulation with the MC momentum corrections applied. The band represents the effect of the systematic uncertainties on the MC momentum corrections. Simulations are normalised to data. Right: Absolute efficiency of the Level 1 (L1) MU20 trigger in 2015 (black) and in 2016 (red) plotted as a function of $\phi$ of offline muon candidates in the barrel detector region. The efficiency is computed with respect to offline isolated reconstructed muon candidates. The MU20 trigger requires that a candidate passed the $20 \mathrm{GeV}$ threshold requirement of the L1 muon trigger system. The efficiency is measured using a tag-and-probe method with $\mathrm{Z} \rightarrow \mu \mu$ candidates, with no background subtraction applied, in $13 \mathrm{TeV}$ data from 2015 and 2016 with $25 \mathrm{~ns}$ LHC bunch spacing. Only statistical data uncertainties are shown.

opposite sign electron-positron pairs from Z-boson decays for 2015 and 2016 as a measure of the energy scale. The 2016 data have been recorded in April 2016 with single colliding bunches or 12 bunch trains. The average number of interactions per bunch crossing was 26. Those are compared to data which were recorded in 2015 with 144 bunch trains and an average number of interactions per bunch crossing of 13. The bunch trains in 2015 and 2016 data have $25 \mathrm{~ns}$ bunch spacing. The same reconstruction software and calibration corrections are applied to both datasets. The 2015 data are normalized to the luminosity of the 2016 data. The difference in the average energy scale from the mass peak position between the 2016 and 2015 datasets is $0.2 \pm 0.1$ (stat)\%. The energy scales in the two datasets are consistent when taking also into account that the early 2016 data with 12 bunch trains (shorter than the drift time in LAr) have a residual pileup systematics estimated to be between 0.05 and $0.1 \%$. The right side of Fig. 4 illustrates the use of calorimeter data in the event selection system, in particular for algorithms searching for significant amounts of missing momentum in a given collision event. Data from trains with both 12 and 72 bunches are used. The events have been selected using single lepton (electron or muon) triggers. The X-axis shows the offline $E_{\mathrm{T}}^{\text {miss }}$ calculated from the sum of electrons, photons and jets, without the contributions from the muons or the track soft term. Three different $E_{\mathrm{T}}^{\text {miss }}$ high-level trigger algorithms are shown: HLT_xe80_tc_lcw_L1XE50 calculates $E_{\mathrm{T}}^{\mathrm{miss}}$ based on calibrated clusters of calorimeter cells, and has a nominal threshold of $80 \mathrm{GeV}$. HLT_xe90_mht_L1XE50 calculates $E_{\mathrm{T}}^{\text {miss }}$ based on reconstructed jets, and has a nominal threshold of $90 \mathrm{GeV}$. HLT_xe100_L1XE50 calculates $E_{\mathrm{T}}^{\text {miss }}$ based on calorimeter cells calibrated at the electromagnetic scale, and has a nominal threshold (at the electromagnetic scale) of $100 \mathrm{GeV}$. All three algorithms are seeded by a L1 trigger algorithm 
with a nominal threshold of $50 \mathrm{GeV}$ which is also shown.

During the shutdown the first arm of Roman-pot detectors of the new ATLAS Forward Proton (AFP) detector was installed and integrated into the ATLAS data acquisition system. The first recorded hit patterns show the expected behaviour, demonstrating that all elements of the detectors are working well. The second arm of the AFP will be installed in the coming 2016/17 shutdown. The other forward detector components of ATLAS are also fully operational.

In summary, the ATLAS detector is in very good shape for 2016 data taking, with close to all channels operational, an already very advanced understanding of the detector performance, and a high data taking efficiency.
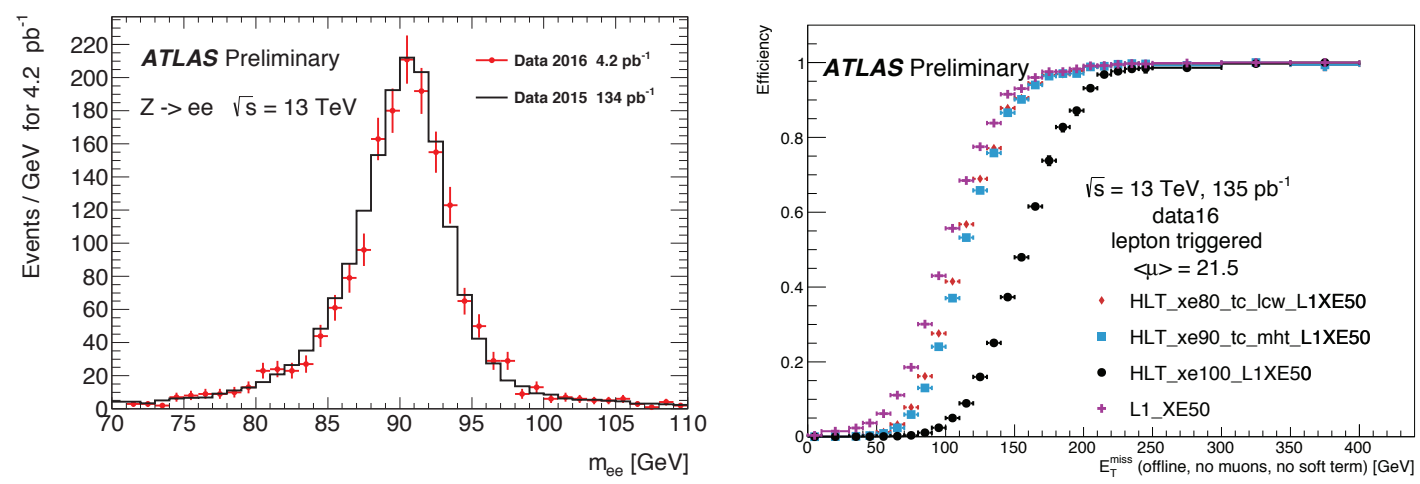

Figure 4: Left: Invariant mass distribution of opposite sign electron-positron pairs from $Z$-boson decays. Electrons or positrons are required to satisfy $E_{\mathrm{T}}>25 \mathrm{GeV},|\eta|<2.37$, excluding the barrel end-cap transition region $(1.37<|\eta|<1.56)$. Right: Efficiency as a function of offline $E_{\mathrm{T}}^{\text {miss }}$ for three different $E_{\mathrm{T}}^{\text {miss }}$ trigger algorithms, using early pp collision data from 2016.

\section{Recent physics highlights}

ATLAS is continuously producing a large number of scientific results, only a selection can be mentioned here. While the analysis of Run 2 data sets now dominates, there are still a few analyses being finalised that take advantage of the large and well understood Run 1 data set at $\sqrt{s}=7$ and $8 \mathrm{TeV}$. One such example is the study of the rare decays of $B_{s}^{0}$ and $B^{0}$ into muon pairs recently published in [2]. Flavour-changing neutral-current (FCNC) processes are highly suppressed in the Standard Model (SM), and their study is relevant to indirect searches for physics beyond the SM. The branching fractions of the decays $B_{s}^{0}$ and $B^{0}$ to $\mu^{+} \mu^{-}$are of particular interest because of the additional helicity suppression and since they are accurately predicted in the SM. The results obtained by ATLAS are consistent with the SM within $2.0 \sigma$. The data are however still consistent with zero for these rare branching fractions. A further example of a recently published analysis based on Run 1 data is the precision measurement of the top-quark mass [3], measured in the $t \bar{t} \rightarrow$ dilepton channel from $20.2 \mathrm{fb}^{-1}$ of $\sqrt{s}=8 \mathrm{TeV}$ proton-proton collision data. Compared to the previous ATLAS measurement in this decay channel, the event selection has been refined exploiting the average $p_{\mathrm{T}}$ of the lepton-b-jet pairs to enhance the fraction of correctly reconstructed events, reducing the systematic uncertainties. The measured value is $m_{\text {top }}=172.99 \pm 0.41$ (stat) \pm 
0.74 (syst) $\mathrm{GeV}$, with a total uncertainty of $0.84 \mathrm{GeV}$. The precision is mainly limited by systematic uncertainties, mostly by the calibration of the jet energy scale, to a lesser extent by the calibration of the b-jet energy scale relative to that of light jets, and by the Monte Carlo modelling of signal events. This measurement is combined with the ATLAS measurements in the $t \bar{t} \rightarrow$ lepton+jets and $t \bar{t} \rightarrow$ dilepton decay channels from $\sqrt{s}=7 \mathrm{TeV}$ data [4]. The combination of the three measurements, taking correlations into account, results in $m_{\text {top }}=172.84 \pm 0.34$ (stat) \pm 0.61 (syst) $\mathrm{GeV}$, with a total uncertainty of $0.70 \mathrm{GeV}$, i.e. a relative precision of $0.4 \%$.
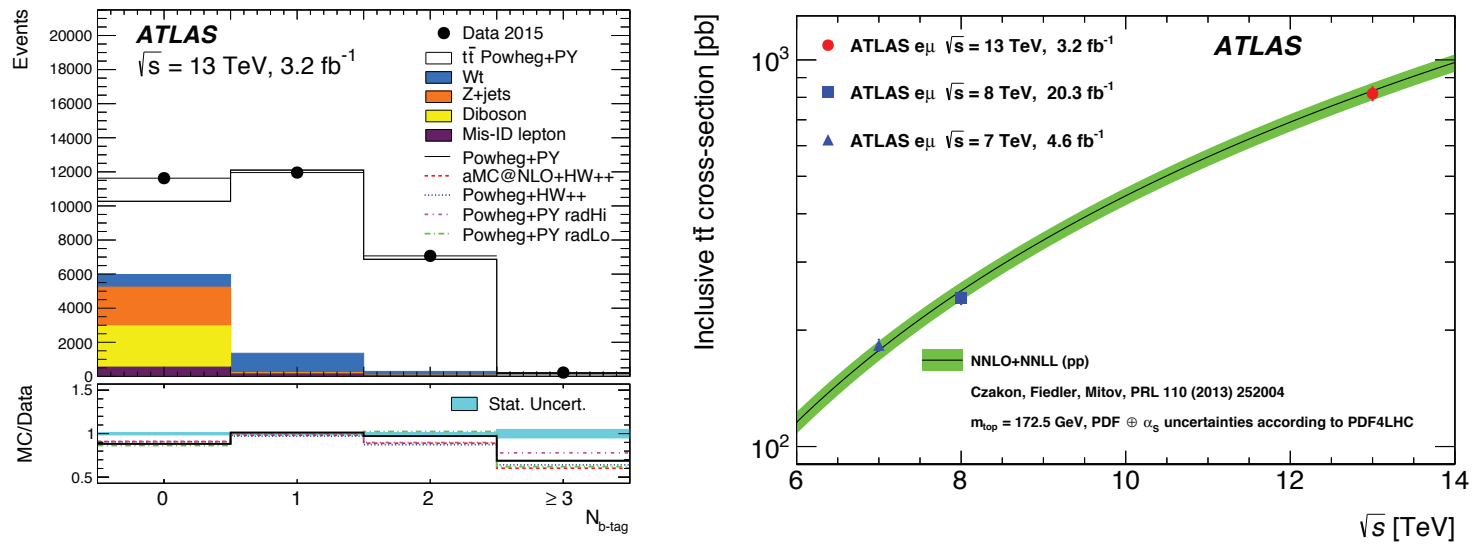

Figure 5: Left: Distribution of the number of b-tagged jets in preselected opposite-sign $e \mu$ events [5]. The data are shown compared to the prediction from simulation, broken down into contributions from $t \bar{t}$ (using the baseline Powheg+Pythia6 sample), Wt single top, Z+jets, di-bosons, and events with fake electrons or muons, normalised to the same integrated luminosity as the data. The lower part of the figure shows the ratio of simulation to data, using various $t \bar{t}$ signal samples, and the cyan band indicates the statistical uncertainty. The $t \bar{t}$ contribution is normalised to the theoretical $t \bar{t}$ cross-section prediction at $\sqrt{s}=13 \mathrm{TeV}$ of $832 \mathrm{pb}$. Right: Cross-section for $t \bar{t}$ pair production in $p p$ collisions as a function of centre-of-mass energy [5]. ATLAS results in the di-lepton $e \mu$ channel at $\sqrt{s}=13,8$ and $7 \mathrm{TeV}$ are compared to the NNLO+NNLL theoretical predictions.

Moving on to Run 2 analyses, ATLAS has recently published a measurement of the $t \bar{t}$ production cross-section using $e \mu$ events with b-tagged jets [5]. This measurement follows the methodology of the most precise measurement obtained in Run 1 [6]. It uses events with an oppositecharge electron-muon pair in the final state. Jets containing b-quarks are tagged using an algorithm based on track impact parameters and reconstructed secondary vertices. The numbers of events with exactly one and exactly two b-tagged jets are counted and used to determine simultaneously the $t \bar{t}$ cross-section and the effective efficiency to reconstruct and b-tag a jet from a top-quark decay, thereby minimising the associated systematic uncertainties. Fig. 5 (left) shows the number of b-tagged jets in preselected opposite-sign $e \mu$ events, which is modelled well by the simulation. The right side of Fig. 5 shows the cross-section result at $\sqrt{s}=13 \mathrm{TeV}$ together with the ATLAS results of [6], and compared to theoretical predictions, which are in excellent agreement with the measurements. The cross section at $\sqrt{s}=13 \mathrm{TeV}$ is measured to be $\sigma_{t \bar{t}}=$ $818 \pm 8$ (stat) \pm 27 (syst) \pm 19 (lumi) \pm 12 (beam) pb, using the latest luminosity uncertainty of $2.1 \%$. A fiducial measurement corresponding to the experimental acceptance of the leptons is also available. 

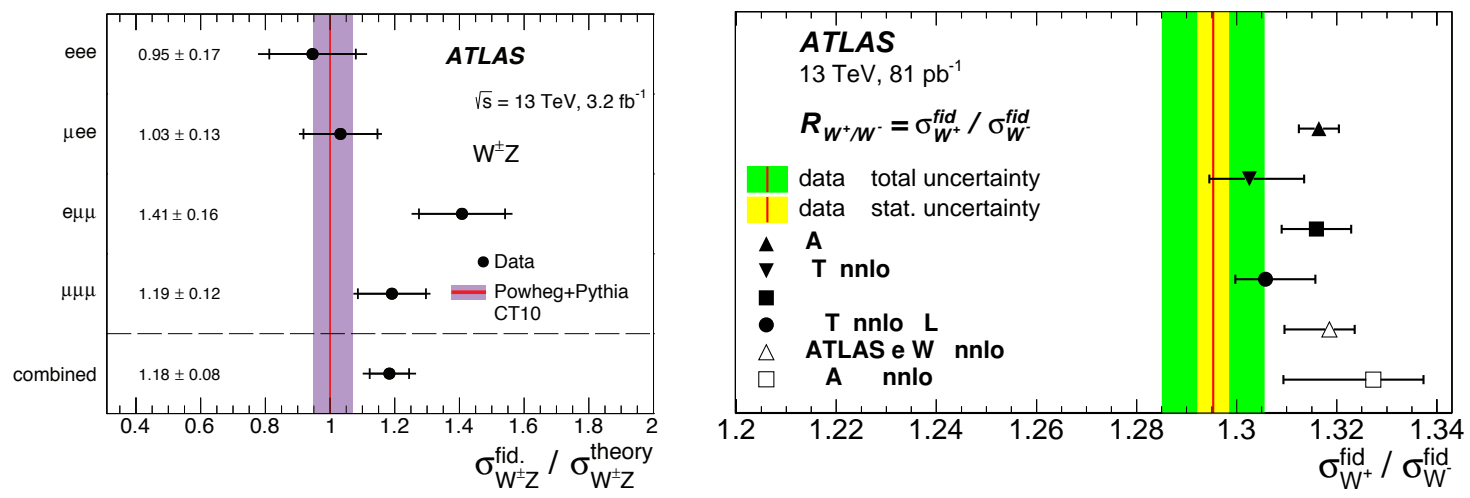

Figure 6: Left: Ratio of the measured $W^{ \pm} Z$ integrated cross sections in the fiducial phase space to the NLO SM prediction from Powheg+Pythia in each of the four three-lepton channels and for their combination [7]. The inner and outer error bars on the data points represent the statistical and total uncertainties, respectively. The NLO SM prediction from Powheg+Pythia using the CT10 PDF set is represented by the red line; the shaded violet band is the total uncertainty in this prediction. Right: Ratio (red line) of $W^{+}$to $W^{-}$boson production cross sections in the fiducial region compared to predictions based on different PDF sets [8]. The inner (yellow) shaded band corresponds to the statistical uncertainty while the outer (green) band shows statistical and systematic uncertainties added in quadrature. The theory predictions are given with only the corresponding PDF uncertainties shown as error bars.

A wealth of measurements involving $W$ and $Z$ bosons has been produced by ATLAS. Recent additions include new measurements of $W^{ \pm} Z$ pair production [7] and $W$ and $Z$ cross sections, and then ratios [8], at $\sqrt{s}=13 \mathrm{TeV}$. The production of $W^{ \pm} Z$ pairs in hadron collisions is an important test of the electroweak sector of the Standard Model. The $W^{ \pm} Z$ final states arise from two vector bosons radiated by quarks or from the decay of a virtual $W$ boson into a $W^{ \pm} Z$ pair, which involves a triple gauge coupling (TGC). In addition, $W^{ \pm} Z$ pairs can be produced in vector-boson scattering processes, which involve triple and quartic gauge couplings (QGC) and are sensitive to the electroweak symmetry breaking sector of the SM. New physics could manifest in $W^{ \pm} Z$ final states as a modification of the TGC and QGC strength. Precise knowledge of the $W^{ \pm} Z$ production cross section is therefore necessary in the search for new physics. Fig. 6 (left) [7] shows the measured $W^{ \pm} Z$ integrated cross sections in the fiducial phase space closely matching the detector acceptance compared to the NLO SM prediction. The measured combined cross section is higher than the SM NLO prediction. However, given that recent calculations in NNLO for the total cross section yield larger values, it is likely that this difference will decrease as soon as NNLO calculations are available for the fiducial phase space.

The measurements of cross-section ratios [8] benefit from the cancellation of many systematic uncertainties, and are therefore powerful tools to constrain PDF fits. In particular, the fiducial cross-section ratio of $W^{+}$to $W^{-}$, measured with an uncertainty of $0.8 \%$, is able to discriminate amongst the various PDF predictions as shown in Fig. 6 (right). These results form a basis for further tests of perturbative QCD and exploration of the partonic content of the proton.

While there was not enough data available in time for the conference to re-establish the SM Higgs signal for $\sqrt{s}=13 \mathrm{TeV}$, or to study anomalies such as the excess observed in the di-photon mass spectrum in 2015 [9] in more detail, many searches taking advantage of the $13 \mathrm{TeV}$ centre- 

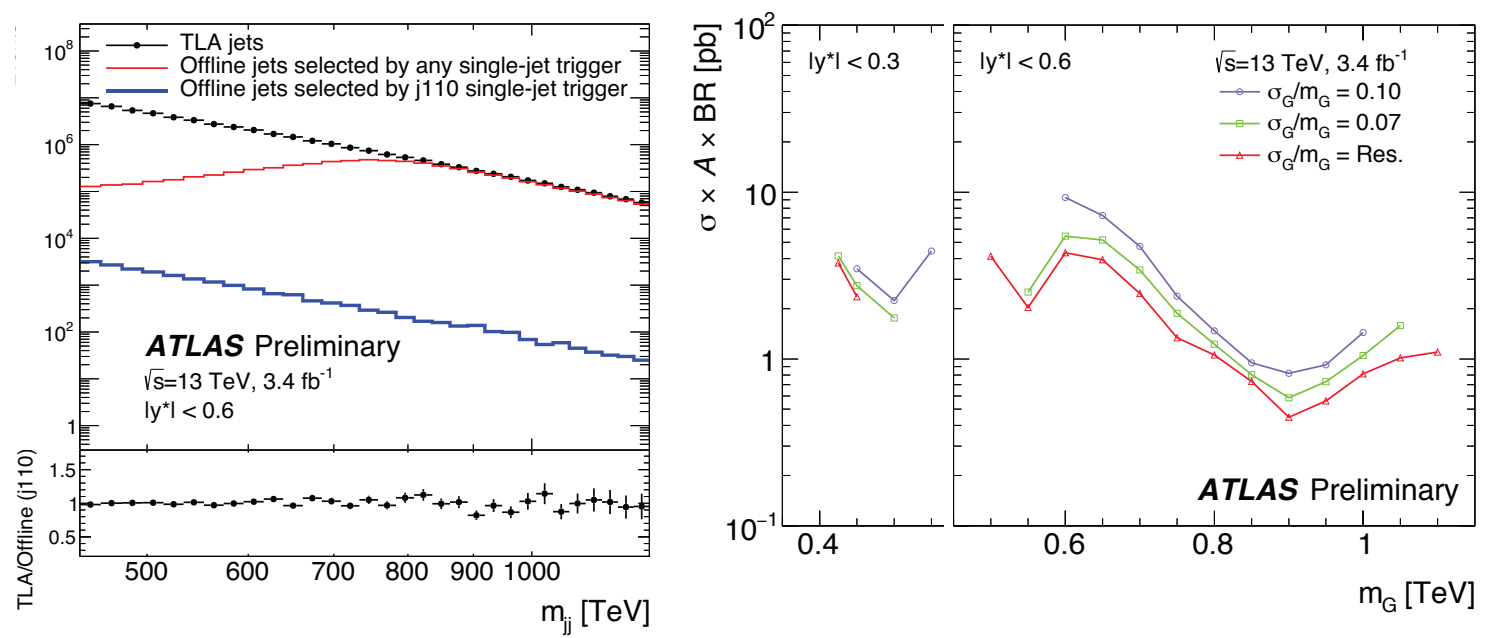

Figure 7: Left: Comparison between the data used by the trigger-object level analysis and the data selected by using an OR of any single jet trigger [10]. The ratio plot compares the shape of the dijet mass distribution of TLA trigger jets and offline jets collected using the HLT_j110 trigger, normalised to the same integral as the trigger-level spectrum. The average prescale factor for the HLT_j110 trigger is 2300. Right: The 95\% credibility-level observed upper limits on cross-section times acceptance times branching ratio $(\sigma \times A \times B R)$ to two jets, for a hypothetical signal producing a Gaussian contribution to the observed $m_{j j}$ distribution, with a width varying from the detector mass resolution to $10 \%$ of its mass [10].

of-mass energy had already been performed, constraining the available phase space for a variety of signatures beyond the SM. One example is the search for light dijet resonances using a triggerobject level analysis (TLA) [10], targeting low-mass dijet resonances between $450 \mathrm{GeV}$ and 950 $\mathrm{GeV}$. Searches for dijet resonances with sub-TeV masses using the ATLAS detector are statistically limited by the bandwidth available to inclusive single-jet triggers. Due to large SM multi-jet backgrounds, these triggers must be prescaled to record full events at a manageable rate. However, one can avoid this limitation by recording only the subset of information in each event needed for such a search, allowing much higher trigger rates. The effect can be seen in Fig. 7 (left), which compares the data used by the trigger-object level analysis and the data selected by using an OR of any single jet trigger as a function of the dijet mass distribution. The figure demonstrates the increase in statistics at lower dijet masses achieved by the TLA strategy as well as the agreement between the shapes of the distributions of jets reconstructed in the trigger and jets reconstructed offline. No excesses are found, and limits are set on Dark Matter mediators with axial vector couplings to quarks and Dark Matter particles, and on generic Gaussian resonances. Fig. 7 (right) shows the $95 \%$ credibility-level observed upper limits on cross-section times acceptance times branching ratio $(\sigma \times A \times B R)$ to two jets, for a hypothetical signal producing a Gaussian contribution to the observed $m_{j j}$ distribution. The limits are shown as a function of the mean mass of the Gaussian distribution, $m_{G}$, for different relative widths $\sigma_{G} / m_{G}$. The smallest relative width shown ('Res') corresponds to a width $\sigma_{G}$ equal to the detector mass resolution. This relative width is between $4 \%$ and $6 \%$ for high and low $m_{j j}$ values, respectively. Results are also shown for larger relative widths of $7 \%$ and $10 \%$. For all mass points to which the nominal $\left|y^{\star}\right|<0.6$ distribution is sensitive, it is selected to define the limit shown. Lower mass points accessible only to the $\left|y^{\star}\right|<0.3$ spectrum 
are shown on the left. Here $y^{\star}$ is defined as half the difference in rapidities of the two jets. A range of mass points spanning $[425 \mathrm{GeV}, 1100 \mathrm{GeV}]$ are thus covered using the combination of the two spectra. While the y axis is shared between the two selections, the signal acceptance varies, thus the two sets of limit points relate to two different interpretations of $\sigma \times A \times B R$. This analysis excludes at $95 \%$ credibility-level $Z^{\prime}$ signals and cross-sections for new processes that would produce a Gaussian contribution to the dijet mass distribution. Gaussian contributions with effective cross-sections ranging from approximately $3 \mathrm{pb}$ at $450 \mathrm{GeV}$, to $9 \mathrm{pb}$ at $600 \mathrm{GeV}$, and to $0.7 \mathrm{pb}$ at $850 \mathrm{GeV}$ are excluded.

\section{Conclusions}

By the time of the conference ATLAS had been already taking data in the 2016 LHC run for several weeks with high data quality and data taking efficiency. Much of the detector performance for 2016 had already been established and is continuously being studied further and improved. In addition to the detector systems the computing and software areas are also operating very well. There is a steady output of physics results, with further important Run 1 results still being produced, and results based on Run 2 data increasingly taking over.

\section{References}

[1] ATLAS Collaboration, The ATLAS Experiment at the CERN Large Hadron Collider, JINST 3 (2008) S08003.

[2] ATLAS Collaboration, Study of the rare decays of $B_{s}^{0}$ and $B^{0}$ into muon pairs from data collected during the LHC Run 1 with the ATLAS detector, submitted to Eur. Phys. J. C., arXiv:1604.04263 [hep-ex].

[3] ATLAS Collaboration, Measurement of the top quark mass in the $t \bar{t} \rightarrow$ dilepton channel from $\sqrt{s}=8$ TeV ATLAS data, Physics Letters B 761 (2016) 350, arXiv:1606.02179 [hep-ex].

[4] ATLAS Collaboration, Measurement of the top quark mass in the $t \bar{t} \rightarrow$ lepton + jets and $t \bar{t} \rightarrow$ dilepton channels using $\sqrt{s}=7 \mathrm{TeV}$ ATLAS data, Eur. Phys. J. C (2015) 75, arXiv:1503.05427 [hep-ex].

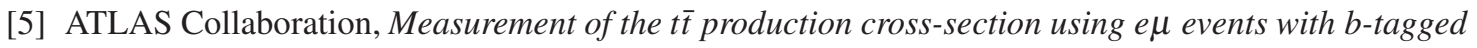
jets in pp collisions at $\sqrt{s}=13$ TeV with the ATLAS detector, Phys. Lett. B761 (2016) 136, arXiv:1606.02699 [hep-ex].

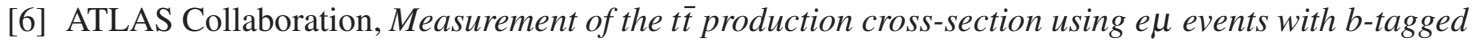
jets in pp collisions at $\sqrt{s}=7$ and 8 TeV with the ATLAS detector, Eur. Phys. J. C 74 (2014) 3109, arXiv:1406.5375 [hep-ex].

[7] ATLAS Collaboration, Measurement of the WZ boson pair-production cross section in pp collisions at $\sqrt{s}=13$ TeV with the ATLAS Detector, Phys. Lett. B 762 (2016) 1, arXiv:1606.04017 [hep-ex].

[8] ATLAS Collaboration, Measurement of $W^{ \pm}$and Z-boson production cross sections in pp collisions at $\sqrt{s}=13$ TeV with the ATLAS detector, Phys. Lett. B 759 (2016) 601, arXiv:1603.09222 [hep-ex].

[9] ATLAS Collaboration, Search for resonances in diphoton events at $\sqrt{s}=13 \mathrm{TeV}$ with the ATLAS detector, JHEP 09 (2016) 001, arXiv:1606.03833 [hep-ex]. 
[10] ATLAS Collaboration, Search for light dijet resonances with the ATLAS detector using a Trigger-object Level Analysis in LHC pp collisions at $\sqrt{s}=13 \mathrm{TeV}$, ATLAS-CONF-2016-030, 2016, URL: https://cds.cern.ch/record/2161135. 